\title{
Adult-Onset Epilepsy in Klinefelter Syndrome? Cognitive and Neurophysiological Evaluation of a 56-Year-Old Man
}

\author{
Yvonne Höller ${ }^{1,2^{*}}$ Adriana Sciarrone ${ }^{2}$, Aljoscha Thomschewski ${ }^{2,3,4}$ and Nathalie Gerner,3 \\ ${ }^{1}$ Faculty of Psychology, University of Akureyri, Iceland \\ ${ }^{2}$ Department of Neurology, Christian Doppler Medical Centre and Centre for Cognitive Neuroscience, \\ Paracelsus Medical University, Austria \\ ${ }^{3}$ Department of Mathematics, Paris-Lodron University of Salzburg, Austria \\ ${ }^{4}$ Spinal Cord Injury and Tissue Regeneration Center Salzburg, Paracelsus Medical University Salzburg, \\ Austria
}

*Corresponding author: Dr. Yvonne Höller, Dipl.-Ing., Faculty of Psychology, University of Akureyri, Norðurslóð 2, 600 Akureyri, Iceland; Department of Neurology, Christian Doppler Medical Centre and Centre for Cognitive Neuroscience, Paracelsus Medical University, Ignaz Harrer Str. 79, 5020 Salzburg, Austria

\begin{abstract}
Klinefelter syndrome (KS) is one of the most common sex chromosome abnormalities and several cases are documented where children with KS present with epilepsy. Also, cognitive function deficits are common, despite high-functioning cases might occur. However, most reported cases are children or young adults.

We report a case of a 56-year-old patient with KS who presented in our clinic with potentially epileptic events. We found left-lateralized temporal intermittent rhythmic delta in routine electroencephalogram (EEG), high-frequency oscillations (HFOs, > $80 \mathrm{~Hz}$ ) in high-density EEG (HD-EEG) that where pronounced over the left hemisphere, as well as left-temporal and paraventricular structural changes. Despite the patient was cognitively high functioning with excellent verbal and episodic memory performance, executive function tests involving inhibitory skills yielded significant differences against an age and sex-matched control group $(\mathrm{N}=18)$.

This case suggests that high-functioning patients with KS may present with epilepsy-typical activity, that could be indicative for late-onset epilepsy. Diagnostic means of routine EEG can be complemented by HFO analysis of HD-EEG. Normal to high cognitive functioning may co-occur with deficits in single domains such as inhibition.
\end{abstract}

\section{Keywords}

Klinefelter syndrome, Late-onset epilepsy, Cognitive function, Electroencephalography

\begin{abstract}
Abbreviations
KS: Klinefelter's Syndrome; HFOs: High-Frequency Oscillations; EEG: Electroencephalogram; HD-EEG: High-Density Electroencephalogram, EMU: Epilepsy Monitoring Unit
\end{abstract}

\section{Introduction}

Klinefelter syndrome (KS) is one of the most common sex chromosome abnormalities, occurring in up to one in 500 male births [1]. Typical characteristics of KS are hypogenitalism and hypogonadism, long limbs, tall and slim stature in childhood, and decreased facial and pubic hair [2]. Several structural brain imaging studies in patients with KS have shown reduced brain volume, mainly in frontal and temporal lobes and altered cognitive functioning [3,4].

Among patients with $\mathrm{X}$ chromosome anomalies, patients with KS are particularly vulnerable to epilepsy [5]. The EEG in patients with KS and epileptic seizures shows both focal and generalized epileptiform discharges [1]. The onset of epilepsy in KS was reported to be in childhood, adolescence, or latest in early adulthood (21 years), and always in cases with mild to severe mental retardation [1,6-9].

We report a case of KS which shows that epilepsy in KS can occur:

\footnotetext{
Citation: Höller Y, Sciarrone A, Thomschewski A, Gerner N (2019) Adult-Onset Epilepsy in Klinefelter Syndrome? Cognitive and Neurophysiological Evaluation of a 56-Year-Old Man. Int J Rare Dis Disord 2:009. doi.org/10.23937/2643-4571/1710009

Accepted: September 04, 2019; Published: September 06, 2019

Copyright: (C) 2019 Höller Y, et al. This is an open-access article distributed under the terms of the Creative Commons Attribution License, which permits unrestricted use, distribution, and reproduction in any medium, provided the original author and source are credited.
} 
1) In the absence of mental retardation.

2) In adulthood (42 years).

We provide a detailed analysis of EEG epileptiform abnormalities, structural changes, and a cognitive and behavioral evaluation against a control group. In addition, high-frequency oscillations (HFO) above $80 \mathrm{~Hz}$ are suggested to be a potential marker for pathological changes in epilepsy [10]. For the first time, we examine HFOs in a patient with KS and epilepsy.

\section{Material and Methods}

A 56-year-old man with KS presented with potentially epileptic events with an onset at the age of 42 . The patient was admitted at the Epilepsy Monitoring Unit (EMU) of the Department of Neurology, Christian Doppler Medical Center, Salzburg. The patient was enrolled into a study about HFOs in the scalp-EEG in epilepsy. The study protocol was approved by the Ethics Commission Salzburg (415-E/1806/17-2018).

We performed standard video EEG at $256 \mathrm{~Hz}$ for four days and high-density EEG (HD-EEG) at $1000 \mathrm{~Hz}$ for 2 hours during which cognitive tasks were performed. We compared the patient's cognitive performance in these tasks to a control group of 18 males, on average 58.17 years old $($ median $=55 ; S D=8.81)$, all right handed.

The patient and the control group were screened for depression and chronotype. For detailed information on cognitive tests, evaluation of behavioral data, the control group, and HFO analysis, see the Supplementary Section: Methods.

We reported means, standard deviations and medians for the control groups. We conducted one-sample t-tests to assess the significant deviation of the patient from the sample, using Bonferroni-correction for multiple comparisons.

\section{Results}

\section{Case description}

The 56-year-old KS patient reported seizure onset at the age of 42 . Description of events was provided by the patient and witnesses. The patient experienced two types of seizures.

A total of five events of Type 1 was described by the patient as a sudden complete loss of control over the left side of the body. The patient reported no pain in head or muscles, no loss of memory, no post-ictal cognitive symptoms, no vomitus or nausea, no secessus and no tongue biting. The events occurred only during wakefulness. Neurological evaluation concluded focal seizures with sensomotoric symptoms on the left side without loss of consciousness. The onset was presumed to be right frontal.

Furthermore, the patient reported one brief episode of Type 2: Loss of consciousness without prodromal symptoms; no convulsions were observed. After regaining awareness, the patient was fully reactive and orientated. The event occurred 3 months prior to admission to the EMU.

KS was diagnosed at the age of 29 years. Further diagnoses of the patient were: microadenoma of the pituitary gland; diabetes mellitus Type 2, obesity II; hypercholesterolemia, concentric left ventricular hypertrophy, COPD II, non-alcoholic fatty liver disease, osteoporosis. The patient was under medication with Trittico ret. $150 \mathrm{mg} \mathrm{0-0-0-2/3}$ and testosterone supplementation.

The neurological examination was normal except for a discrete hypoesthesia at the level of T5/T6 on the left. The examination concluded a normal degree of cognitive functioning. Psychological examination indicated a light depression (Beck's Depression Inventory score of 17) and neutral circadian type. The patient had a polytechnic degree and attended the university of applied sciences, which supports the impression of a high-functioning case of KS. No standardized test for intelligence was conducted.

\section{MRI findings}

MRI (see Figure 1) revealed a moderate symmetrical expansion of the cerebrospinal fluid spaces. The left hippocampal gyrus appeared to be discretely flattened.

Single residual vascular lesions could be seen periventricular in the anterior parts of cella media on both sides, and a single periventricular lesion in the posterior part of the left cella media.

\section{EEG findings}

The five-day EMU video EEG showed a well-formed 9-11 Hz posterior alpha rhythm, with an amplitude of 25-30 $\mu \mathrm{V}$. This was symmetrically distributed over both temporo-occipital regions, it was synchronous and attenuated normally on eye opening. Superimposed beta activity $12-20 \mathrm{~Hz}$ was seen with a widespread distribution. Normal EEG sleep phenomena were observed (Sleep spindles, Vertex waves, K-complexes). Intermittent rhythmical delta activity (TIRDA) [11] was noted over the left temporal regions, maximum on $\mathrm{F} 7 / \mathrm{T} 7$, with a duration up to 15 seconds.

The 2-hour HD-EEG recording showed again 9-10 Hz posterior alpha rhythm with superimposed widespread beta activity. During ten-minute resting, short periods of drowsiness were recorded. Early Vertex waves could be noted over the $\mathrm{Cz}$ channel. Within 5 min of HD-EEG, 18 HFO-like artefacts and 10 HFO-like events were detected, among which one event could be classified as potential HFO, recorded during rest over the left Centro-parietal region (see Supplementary Section: Results, Figures 4 and Figure 5). On this channel, a total of three HFO-like events were detected. No seizures were recorded, neither in the video EEG nor in the HD-EEG. 


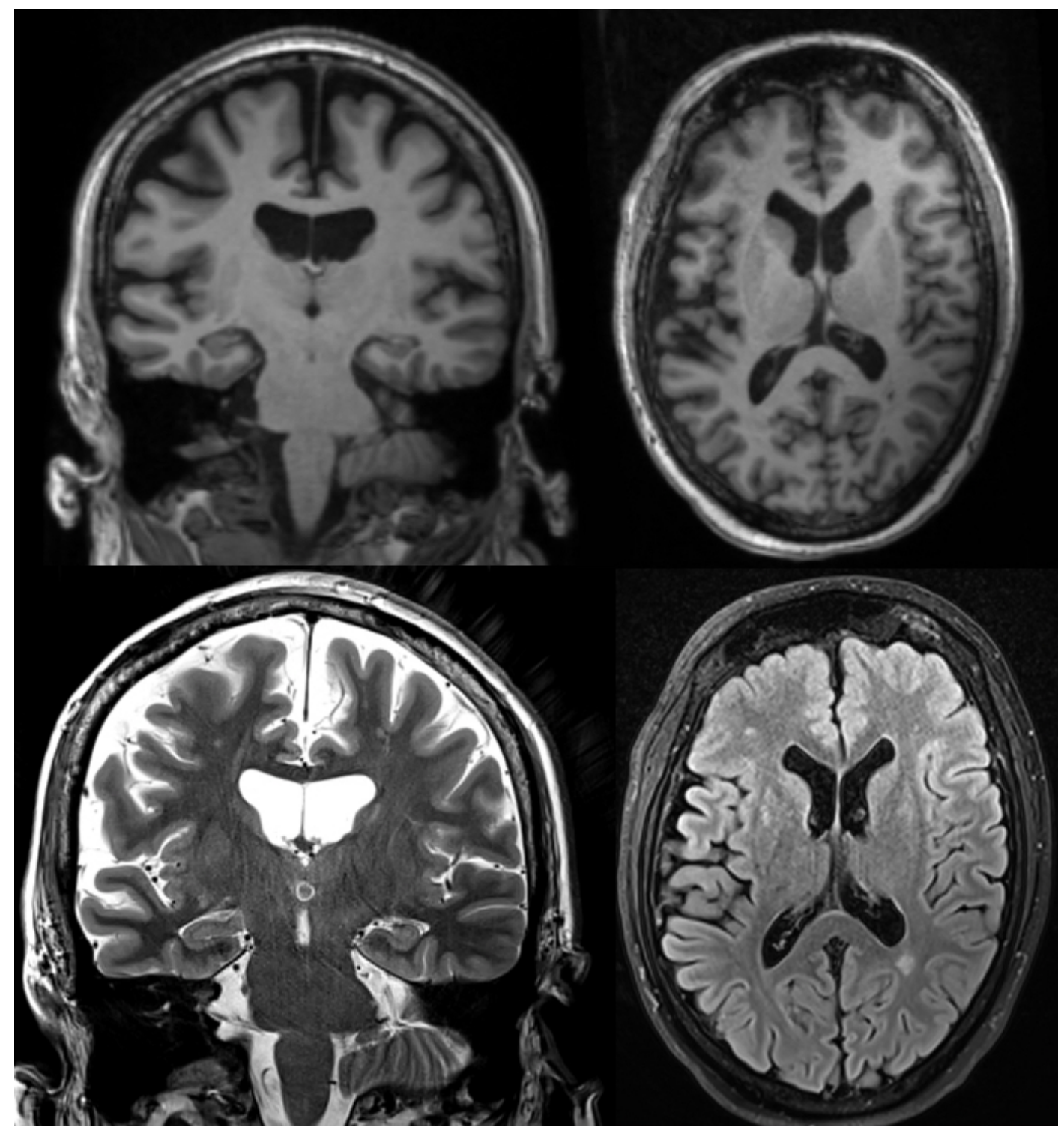

Figure 1: Structural magnetic resonance imaging. Top T1 MP-rage; Bottom left: T2 hippocampal slice; Bottom right: T2 flair;

\section{Behavioral findings}

Table 1 shows the behavioral results of the patient and the control group. The patient was distracted during the afternoon bedside-session by medical staff interfering with the testing, which is most likely to explain a worse performance in the second version of the tests. From those test results that were significant over both sessions we concluded that the patient's performance was significantly better for free recall of episodic memory elements (Virtual Town recall). However, the accuracy during procedural learning (Finger Tapping) was significantly worse, which is a sign of deficient inhibition. Although not replicable at the strict level of significance, the accuracy for inhibition of response $(\mathrm{Go} /$ No-Go) seemed to be impaired $(p=0.01)$.

$\varnothing$ : Mean; SD: Standard Deviation; N: Sample Size; Finger Tapp: Finger Tapping Task; nmcg: Non-Matched Control Group; RT: Reaction Time; "Statistically significant at Bonferroni-corrected level of $p=0.05 / 40=$ 0.0013 .

\section{Discussion}

To our knowledge, the present study demonstrated for the first time a case of potential seizures, and thus, presumed adult-onset epilepsy in a high-functioning patient with KS. According to the criteria of the IIAE [12], the nature of the events needs to be clarified in order to diagnose epilepsy. However, the case can be considered as possible epilepsy.

The case demonstrated a tendency of the known deficits in executive functions that are typical for KS $[3,13]$ in the Go/No-go test and significant difficulties with inhibition as demonstrated by lower accuracy during procedural learning. Executive function problems in context of KS are not uniform but certain domains may be affected more than others [3]. Future studies should conduct standardized tests in order to challenge these assumptions.

The most prominent gray matter volume reductions in KS are found in temporal and frontal lobe regions [14]. The abnormal left-temporal pattern in the stan- 
Table 1: Behavioral data and test results from one-sample t-test.

\begin{tabular}{|c|c|c|c|c|c|c|}
\hline \multirow[b]{2}{*}{ Task } & \multicolumn{4}{|c|}{ Control group } & \multirow[t]{2}{*}{ Case } & \multirow{2}{*}{ One-sample t-test results } \\
\hline & $\varnothing$ & SD & Med & $\mathbf{N}$ & & \\
\hline Word Pair 1 recall & 13.61 & 9.03 & 14.5 & 18 & 13 & $t(17)=0.28 ; p=0.78$ \\
\hline Word Pair 2 recall & 14.06 & 7.46 & 15.5 & 16 & 3 & $t(15)=5.74 ; p=0.00003^{*}$ \\
\hline Word Pair 1 recognition nmcg & 49.5 & 6.79 & 52 & 10 & 52 & $T(9)=-1.11 ; p=0.30$ \\
\hline Word Pair 2 recognition nmcg & 53 & 4.53 & 53 & 8 & 40 & $t(7)=7.60 ; p=0.0001^{*}$ \\
\hline Virtual Town 1 recognition & 5.5 & 3.15 & 6 & 18 & 6 & $T(17)=0-0.65 ; p=0.52$ \\
\hline Virtual Town 2 recognition & 6.35 & 3.85 & 7 & 17 & 8 & $T(16)=-1.71 ; p=0.11$ \\
\hline Virtual Town 1 recall & 7.73 & 3.41 & 8 & 15 & 14 & $T(14)=-6.87 ; p=0.000008^{*}$ \\
\hline Virtual Town 2 recall & 8 & 3.31 & 8 & 13 & 12 & $T(12)=-4.19 ; p=0.001^{*}$ \\
\hline Virtual Town 1 details & 9.6 & 3.02 & 8 & 15 & 16 & $T(14)=-4.04 ; p=0.001^{\star}$ \\
\hline Virtual Town 2 details & 8.77 & 4.66 & 8 & 13 & 14 & $T(12)=-3.89 ; p=0.002$ \\
\hline Virtual Town 1 time & 3.6 & 1.62 & 4 & 15 & 5 & $T(14)=-3.22 ; p=0.006$ \\
\hline Virtual Town 2 time & 4.38 & 2.5 & 4 & 13 & 4 & $T(12)=0.053 ; p=0.60$ \\
\hline Virtual Town 1 allocentric & 3 & 2.92 & 3 & 15 & 4 & $T(14)=-1.28 ; p=0.22$ \\
\hline Virtual Town 2 allocentric & 2.92 & 1.69 & 3 & 13 & 5 & $\mathrm{~T}(12)=-4.27 ; \mathrm{p}=0.001^{\star}$ \\
\hline Virtual Town 1 egocentric & 5.07 & 2.29 & 5 & 15 & 6 & $T(14)=-1.52 ; p=0.15$ \\
\hline Virtual Town 2 egocentric & 5.53 & 1.86 & 6 & 13 & 4 & $T(12)=2.86 ; p=0.01$ \\
\hline Finger Tapp 1 speed sec, training & 9.02 & 6.10 & 7.15 & 14 & 5.59 & $t(13)=2.03 ; p=0.06$ \\
\hline Finger Tapp 1 speed sec, performance & 7.17 & 4.47 & 7 & 14 & 6.31 & $t(13)=1.50 ; p=0.16$ \\
\hline Finger Tapp 2 speed sec, training & 6.79 & 3.48 & 6.62 & 12 & 5.31 & $t(11)=0.63 ; p=0.54$ \\
\hline Finger Tapp 2 speed sec, performance & 6.49 & 3.13 & 6.51 & 13 & 4.94 & $t(12)=1.71 ; p=0.11$ \\
\hline Finger Tapp 1 accuracy $\%$, training & 56.78 & 11.12 & 57.32 & 14 & 41 & $t(13)=-1310.9 ; p=1.1186 e-34$ \\
\hline Finger Tapp 1 accuracy $\%$, performance & 91.25 & 5.75 & 92.37 & 14 & 85.9 & $t(13)=-3687,4 ; p=1.6215 e-40$ \\
\hline Finger Tapp 2 accuracy $\%$, training & 79.73 & 19.66 & 82.25 & 13 & 59.69 & $t(12)=-1499.9 ; p=5.1961 e-33$ \\
\hline Finger Tapp 2 accuracy \%, performance & 86.86 & 18.56 & 93.71 & 13 & 68.94 & $t(12)=-1270.8 ; p=3.7977 e-32^{*}$ \\
\hline Stroop 1 accuracy \%, target nmcg & 91.88 & 14.54 & 96.88 & 10 & 100 & $T(9)=-1.68 ; p=0.12$ \\
\hline Stroop 1 accuracy \%, inference nmcg & 92.29 & 15.14 & 97.88 & 10 & 97.93 & $\mathrm{~T}(9)=-1.11 ; p=0.29$ \\
\hline Stroop $1 \mathrm{RT}$ target $(\mathrm{ms}) \mathrm{nmcg}$ & 955 & 246 & 912 & 10 & 974 & $T(9)=-0.23 ; p=0.82$ \\
\hline Stroop $1 \mathrm{RT}$ inference $(\mathrm{ms}) \mathrm{nmcg}$ & 1157 & 355 & 1070 & 10 & 1101 & $T(9)=0.47 ; p=0.65$ \\
\hline Stroop 2 accuracy \%, target nmcg & 98.44 & 4.13 & 100 & 8 & 100 & $T(7)=-1 ; p=0.35$ \\
\hline Stroop 2 accuracy \%, inference nmcg & 94.27 & 4.26 & 94.79 & 8 & 100 & $T(7)=-3.55 ; p=0.009$ \\
\hline Stroop 2 RT target nmcg & 877 & 254 & 857 & 8 & 1186 & $T(7)=-3.22 ; p=0.01$ \\
\hline Stroop 2 RT inference nmcg & 1004 & 352 & 999 & 8 & 1020 & $\mathrm{~T}(7)=-0.12 ; \mathrm{p}=0.91$ \\
\hline Go/No-Go 1 accuracy \%, target nmcg & 74.33 & 30.41 & 88.67 & 10 & 87 & $\mathrm{~T}(9)=-1.22 ; p=0.25$ \\
\hline Go/No-Go 1 accuracy \%, non-target nmcg & 90 & 6.32 & 90 & 10 & 83 & $T(9)=3.16 ; p=0.01$ \\
\hline Go/No-Go 1 RT target nmcg & 369 & 37 & 365 & 9 & 379 & $T(8)=-0.83 ; p=0.43$ \\
\hline Go/No-Go 1 RT non-target nmcg & 307 & 76 & 280 & 9 & 274 & $T(8)=1.21 ; p=0.26$ \\
\hline Go/No-Go 2 accuracy target nmcg & 71 & 39.07 & 91.67 & 10 & 50 & $\mathrm{~T}(9)=1.61 ; p=0.14$ \\
\hline
\end{tabular}

dard EEG evaluation could be interpreted to be in line with the volume alteration on the left side and thus, possibly reflecting a pathological change. The left lateralized potential HFO finding in the HD-EEG might be a further indicator, though should be interpreted carefully. HFOs on the scalp are only rarely identified because they are of small scale and their distinction from background noise and physiological artefacts cannot be done based on their amplitude in the filtered EEG [15]. The procedure we applied is based on a step-wise exclusion of artefactual sources that might explain the phenomenon. Since this is the first HFO report in KS based on a single case, further research is needed in order to examine the speculation that HFOs could be a marker for pathology in patients with KS and epilepsy.

This case study is limited by the fact that the patient suffered from a series of other conditions, among which vascular lesions could explain abnormalities in brain function. At this point it is impossible to disentangle the effect of KS from other potential causes. Future EEG-studies with adult patients with KS should clarify 
the prevalence of epileptiform activity and HFOs in this population.

\section{Conclusion}

The present study demonstrated a rare case of potential seizures with presumed late-onset epilepsy in a high-functioning patient with KS. This is supported by concordant findings in routine EEG and structural evaluation of MRI. HFO analysis provided further indication. This case emphasizes the importance of splitting up the wide range of executive functions in sub-domains when examining deficits in patients with $\mathrm{KS}$, especially in patients who show high or normal performance in other cognitive domains.

\section{Acknowledgments}

We thank the patient for his participation and the monitoring team of the Epilepsy Monitoring Unit in Salzburg for their support.

\section{Disclosure of Funding}

The presented research was funded by the Austrian Science Fund (FWF): KLI657-B31 and by PMU-FFF: A-18/01/029-HÖL.

\section{References}

1. Tatum IV WO, Passaro EA, Elia M, Guerrini R, Gieron M (1998) Seizures in Klinefelter's syndrome. Pediatr Neuro 19: $275-278$

2. Lanfranco F, Kamischke A, Zitzmann M, Nieschlag E (2004) Klinefelter's syndrome. The Lancet 364: 273-283.

3. Kompus K, Westerhausen R, Nilsson L-G, Hugdahl K, Jongstra $S$, et al. (2011) Deficits in inhibitory executive functions in Klinefelter (47, XXY) syndrome. Psychiatry Res 189: 135-140.

4. Boone K, Swerdloff R, Miller B, Geschwind D, Razani J, et al. (2011) Neuropsychological profiles of adults with Klinefelter syndrome. J Int Neuropsychol Soc 7: 446-456.

5. Grosso S, Farnetani MA, Di Bartolo RM, Berardi R, Pucci L, et al. (2004) Electroencephalographic and epileptic patterns in $\mathrm{X}$ chromosome anomalies. J Clin Neurophysiol 21: 249-253.

6. Gürkas E, Genc HM, Kilic E (2018) Triple X Syndrome with a Rare Finding: Cleft Palate. J Pediatr Res 5: 100-102.

7. Elia M, Musumeci S, Ferri R, Scuderi C, Del Gracco S, et al. (1995) Seizures in Klinefelter's syndrome: A clinical and EEG study of five patients. Ital J Neurol Sci 16: 231-238.

8. Inoue $\mathrm{H}$, Orita $\mathrm{T}$, Matsushige $\mathrm{T}$, Hasegawa $\mathrm{S}$, Ichiyama $\mathrm{T}$ (2012) Klinefelter's syndrome complicated with West syndrome in a 4-month-old boy. Brain Dev 34: 148-150.

9. Miyamoto A, Kitawaki K, Koida H, Nagao K (1992) Klinefelter's syndrome and epileptic psychosis: A case report Psychiatry Clin Neurosci 46: 61-65.

10. Höller Y, Kutil R, Klaffenböck L, Thomschewski A, Höller PM, et al. (2012) High-frequency oscillations in epilepsy and surgical outcome. A meta-analysis. Front Hum Neurosci 9: 574.

11. Geyer JD, Bilir E, Faught RE, Kuzniecky R, Gilliam F (1999) Significance of interictal temporal lobe delta activity for localization of the primary epileptogenic region. Neurology 52: 202-205.

12. Fisher RS, Acevedo C, Arzimanoglou A, Bogacz A, Cross $\mathrm{JH}$, et al. (2014) ILAE official report: A practical clinical definition of epilepsy. Epilepsia 55: 475-482.

13. Temple CM, Sanfilippo PM (2003) Executive skills in Klinefelter's syndrome. Neuropsychologia 41: 1547-1559.

14. Steinman K, Ross J, Lai S, Reiss A, Hoeft F (2009) Structural and functional neuroimaging in Klinefelter (47, XXY) syndrome: a review of the literature and preliminary results from a functional magnetic resonance imaging study of language. Dev Disabil Res Rev 15: 295-308.

15. Höller P, Trinka E, Höller Y (2018) High-Frequency Oscillations in the scalp electroencephalogram: Mission impossible without computational intelligence. Comput Intell Neurosci. 